\title{
Real-Time Simulation of Minimally-Invasive Surgery with Cutting Based on Boundary Element Methods
}

\author{
Ullrich Meier $^{1,2}$, Carlos Monserrat ${ }^{1}$, Nils-Christian Parr ${ }^{1,2}$, \\ Francisco Javier García ${ }^{1}$, José Antonio Gil ${ }^{1}$ \\ ${ }^{1}$ MedICLab, ETSIA, DEGI, Universidad Politécnica de Valencia, Camino de Vera s/n, \\ E-46022 Valencia, Spain \\ ulme@doctor.upv.es, cmonserr@dsic.upv.es \\ ${ }^{2}$ Institut für Angewandte Mechanik und Bauinformatik, \\ Technische Universität Braunschweig, Spielmannstraße 11, \\ D-38106 Braunschweig, Germany
}

\begin{abstract}
Most deformable models for surgery simulation are quite straightforward to achieve the computational speed required for real time simulation. However, they typically are more adjusted to merely graphical representation needs, i.e. surface-oriented (e.g. mass-spring type models), and neglect mechanical realism, although the simulation of cutting in real time is feasible. Finite element (FEM) based models, in turn, depart from continuum mechanics principles, and therefore are more realistic in many cases. But their volumetric structure is not optimal for graphical representation and in this sense produces excessive data, eventually impeding a real-time simulation of incisions. While parting from the same hypotheses as FEM models, a boundary element (BEM) based algorithm passes the influence of an organ's interior to its surface. Thus, it yields comparable simulation results as the prior, but with less data to process. This enables the consideration in real time of local modifications of the underlying boundary element mesh produced when cutting. Actually, the presented algorithm has been successfully tested to simulate incisions in real time.
\end{abstract}

A deformable model based on boundary element methods (BEM) departs from the same continuum mechanical hypothesis as those derived from finite element methods (FEM) [2]. However, it only requires the discretization of the organ's surface into 2-D elements and the definition of $N$ nodes on their outline, coinciding with the typical graphical representation of 3-D objects by means of polygons joined at vertices. The resulting system of equations of order $3 N$ is of the form

$$
\mathbf{H} \cdot U=\mathbf{G} \cdot P+B,
$$

where $\mathbf{H}$ and $\mathbf{G}$ are square influence matrices, $B$ the body force vector (gravity), and $U$ and $P$ the nodal displacement and traction vectors. At some nodes, the displacement is prescribed by the virtual tool with which the node is in contact, and the resulting traction determines the respective force fed back. The majority of nodes will be free, though, i.e. have a prescribed zero traction. Thus, by exchanging some few columns of $\mathbf{H}$ and $\mathbf{G}$, all unknowns are on the left, while on the right hand side most matrix columns can be neglected, as they are multiplied with zeros. 
In order to significantly accelerate the solving time, even more as $\mathbf{H}$ and $\mathbf{G}$ are fully populated and not symmetric, (1) is multiplied with the inverse of the modified system matrix $\mathbf{H}^{\prime}$, which is obtained during the preparation phase when time is not yet the ruling factor. Thus, the vector of unknowns is isolated on the left hand side, and the solving of the system is immediate for different tool positions. Nevertheless, whenever it comes to a new contact, or an already existing one is lost, the composition of $\mathbf{H}^{\prime}$ and with it its inverse change. But the toggling of some nodes between the two states of "free" and "constrained" only affects a few columns of $\mathbf{H}^{\prime}$. Hence, by resorting to the Woodbury formula [3] for the corresponding modification of $\mathbf{H}^{\mathbf{r}^{-1}}$, the required computations are of the order $O\left(N^{2}\right)$ instead of $O\left(N^{3}\right)$ only.

Cutting, in turn, requires the partitioning of some surface elements and the addition of new elements along the created notch, together with the introduction of the corresponding new nodes. This local re-modeling of the underlying boundary element mesh once more produces changes in a few columns of $\mathbf{H}^{\prime}$, and further leads to some new columns and rows. Again, the Woodbury formula can be employed to modify $\mathbf{H}^{\mathbf{r}^{-1}}$ correspondingly, and the required modifications do not exceed the order $O\left(N^{2}\right)$.

The result is a deformable model that is comparable to FEM-based models as far as its robustness or high velocity and accuracy when simulating quasi-linear deformations are concerned. In contrast to the other, though, the BEM-based model here proposed permits the inclusion of gravity and the simulation of cutting in real time. However, as with the FEM-based models, the results are less satisfying for large deformations and for tissue properties with important non-linear portions.

The BEM-based deformable model has been tested for organs modeled with several hundred nodes on MedICLab's surgery simulator [1]. Being based on a standard PC with Pentium III processor with 450MHz / 128Mbyte RAM and Windows NT 4.0 (service pack 6), this simulator represents a low cost approach to surgery simulation. For standard deformations, the computational cost was of the order of a millisecond. When creating new contacts with virtual tools or loosing existing ones, the cost was of the order of tens of milliseconds, and of a hundred milliseconds when simulating shear cuts. Resuming, the here exposed algorithm represents an important advance with respect to other deformable models, as it combines the accuracy of a continuum mechanics based model with the possibility to perform cuts. Our future work will therefore be centered in parallelizing the algorithm to further accelerate it and extending the model to include more complex, non-linear tissue properties and large deformations.

\section{References}

1. Meier, U., García, F.J., Parr, N.-C., Monserrat, C., Gil, J.A., Grau, V., Juan, M.C., Alcañiz, M.: 3D Surgery Trainer with Force Feedback in Minimally Invasive Surgery. To appear in: Proc. CARS 15, Berlin (2001)

2. Monserrat, C., Meier, U., Alcañiz, M., Chinesta, F., Juan, M.C.: A new approach for the real-time simulation of tissue deformations in surgery simulation. Computer Methods and Programs in Biomedicine, 64(2), 77 $\square 85$ (2001)

3. Press, W.H. et al.: Numerical Recipes in C. 2nd ed. Cambridge University Press, Cambridge (1992) 